\title{
Assessing the human immune response to SARS-CoV-2 variants
}

To the Editor-After a successful spillover, a virus would greatly benefit from better adaptation to the new host. This may be the case for SARS-CoV-2, which only recently became a human virus. After nearly a year in tens of millions of human hosts, important variants of SARS-CoV-2 that are more fit have emerged, and in some places have already become the dominant strain ${ }^{1}$, as an evolutionary advantage is provided to the virus by the combined action of poor accuracy of its RNA genome replication and Darwinian selection of the mutations.

In the first period after a spillover, when the number of recovered (and thus immune) people is negligible, the major advantage is achieved by strains that are being transmitted more efficiently, as has already been demonstrated for at least two SARS-CoV-2 variants ${ }^{1,2}$. Subsequently, when the infection spreads and the number of immune people increases, the virus also benefits from evading naturally induced immunity and thus gaining the chance of infecting the same patient twice (or more times). These variants are of concern in the context of vaccination efficacy, as the virus-dodging natural immunity - can also evade some of the vaccine-induced immunity. Eventually, when the majority of the susceptible population is vaccinated with effective vaccines, the variant better suited for survival in the new host will be one that has the ability to evade the vaccine-induced immunity. This would be a major problem, as such a variant could decrease, and even abolish, the beneficial effects of a broad immunization program.

However, the emergence of a variant that is able to escape vaccine-induced immunity may not necessarily occur. First, not all viruses are equally able to evade the immune response of the host. In order to achieve this goal, the virus must have evolved regions that are required to be very immunogenic and also easily modifiable without loss of viral fitness ${ }^{3}$. Many RNA viruses, despite their great potential variability, have been unsuccessful in escaping vaccine immunity, as they were unable to generate a mutant fit to both replicate and escape from the vaccine-induced immune response (e.g., measles, rubella and many others $)^{4,5}$. In such examples, the vaccines retain their full activity decades after their introduction into the population. On the other hand, some viruses are better equipped for evading vaccine- or disease-induced immunity, as they have evolved immunogenic regions that can be easily mutated without loss of viral replication capacity. As a result, these pathogens can successfully evade the host's immune response. This is the case for influenza A, in which the 'head' of its hemagglutinin protein is immunodominant and even a small number of mutations can allow the virus to evade pre-existing immunity (induced by previous infection or vaccination) without any loss of fitness ${ }^{6}$.

Therefore, even if SARS-CoV-2 were able to generate a variant that escapes vaccine immunity, it should not be taken for granted that this variant would be able to replicate with a fitness similar to that of the initial viral variant from which it was generated. Human immunodeficiency virus (HIV) is the champion of 'variation without loss of fitness' ${ }^{6}$, but this is true only for the regions of the virus that have evolved to escape the immune response. When selective pressure against HIV is imposed on reverse transcriptase or on viral protease through the use of antiviral drugs, the virus is able to generate a drug-resistant mutant, but at a price of a reduced replication capacity that results - in appropriately treated patientsin improvement of the clinical outcome as well as a reduction in viral transmission ${ }^{7}$. SARS-CoV-2 variants able to escape vaccine-induced immunity could replicate less, with a possible reduction in virulence and therefore in disease severity.

However, we cannot rule out the possibility that a SARS-CoV-2 mutant will escape vaccine immunity and retain its fitness and pathogenicity. This is the reason that genomic surveillance of the new variants is crucial and will be even more important once a large part of the population is vaccinated.

Although genomic surveillance has provided evidence that supports the hypothesis that particular mutations increase transmission or inhibit the immune response, study of a virus with engineered point mutations is not optimal. It has been shown that gene-gene interactions are critical to a change in the phenotype of the virus, so study of the variant itself is the best way to determine its immune response . $^{8}$

Understandably, researchers should be able to quickly and efficiently detect escape variants; this can be done in an initial phase by sequencing, but once a variant is identified as being 'of concern', a PCR-based assay should be quickly designed, developed, validated and made available worldwide for more-efficient real-time tracking of the spread of the variant itself. Beyond this critical step, efforts should be made to isolate such variants in cell-culture settings, particularly when these variants are infecting vaccinated people. Isolates of viral variants will thus allow evaluation of whether these can be neutralized by serum derived from a vaccinated population. Such screening would be necessary for immediate assessment of whether the infection of a vaccinated patient is due to failure of the antiviral immune response in the host or failure of the vaccination-induced immune response due to an escape variant. For this purpose, the pseudovirus model is quick and convenient, but a plaque-reduction neutralization assay of 'street isolates' is still the gold standard that should be used in such a critical situation ${ }^{8}$. Furthermore, data provided by the neutralization assay would also provide preliminary information of paramount importance about the in vitro replication of the mutant strain. For this purpose, a large panel of serum samples from vaccinated people representing different populations (different age, sex, etc.) should be readily available to various reference laboratories for quick and precise evaluation of the neutralizing capacity of the vaccine-induced immune response to the new isolate. In addition, where possible, more in-depth $\mathrm{T}$ cell immunity assessments should be included in the analysis.

In taking the data thus collected into account, it should be remembered that lack of neutralization does not necessarily mean lack of protection from disease. Even a minimal amount of antibodies could provide protection from infection in some instances, and the incubation time of COVID-19 gives the immune system the time that is needed for an immunity boost that cannot be evaluated for its protective efficacy in vitro ${ }^{9}$. In fact, for some diseases (e.g., rubella), reinfection can occur but may not be clinically important if it is not accompanied by symptoms, does not make the patient infectious and only results in an immunity boost that is beneficial to the patient ${ }^{4}$. Furthermore, for many respiratory viruses that cause diseases that do not provide lifetime immunity after recovery (e.g., RSV, parainfluenza viruses and others), reinfections are less severe (and less 
infectious) than the initial disease from the first encounter with the virus ${ }^{10,11}$. All these observations support the proposal of careful clinical observation of the disease that may eventually affect vaccinated patients, including monitoring selected at-risk people for asymptomatic infection and infectivity. Ultimately, the most information about immune escape is learned in the clinic, when there is a variant-specific drop-off of efficacy, along with a propensity for reinfection as a function of particular variants. The in vitro assays are expected to correlate with the clinical findings, as surrogate metrics to indicate what will be expected to manifest in patients.

Finally, should SARS-CoV-2 be able to perfectly escape vaccine-induced immunity, rapid modification of mRNA vaccines could provide an immunity boost likely to provide protection against the new viral strains as well. However, it is important to be aware of the existence of and the sequence of such variants in the shortest possible time frame in order to mitigate risk and control the consequences.

The Italian word for concern is 'preoccupazione', which comes from 'occupazione' ('take care') and 'pre' ('in advance'). Simply put, this is what needs to be done. Concern about SARS-CoV-2 variants should not cause panic but instead should prompt efforts to ensure that all the necessary steps for mitigating variant impact are taken in advance through the development of tools that detect and characterize these new strains in a timely and standard manner.
Published online: 1 March 2021

https://doi.org/10.1038/s41591-021-01290-0

References

1. European Centre for Disease Prevention and Control. https:// www.ecdc.europa.eu/sites/default/files/documents/SARS-CoV2-variant-multiple-spike-protein-mutations-United-Kingdom.pdf (20 December 2020).

2. Korber, B. et al. Cell 182, 812-827.e19 (2020).

3. Burton, D. R. Nat. Rev. Immunol. 2, 706-713 (2002).

4. Strebel, P. M. \& Orenstein, W. A. N. Engl. J. Med. 381, 349-357 (2019)

5. Lambert, N., Strebel, P., Orenstein, W., Icenogle, J. \& Poland, G. A. Lancet 385, 2297-2307 (2015).

6. Karlsson Hedestam, G. B. et al. Nat. Rev. Microbiol. 6, 143-155 (2008)

7. Eriksen, J. et al. Infect. Dis. 53, 1-8 (2021).

8. Qiangian, L. et al. Rev. Med. Virol. 28, e1963 (2018).

9. Tuaillon, E. et al. J. Immunol. Methods 315, 144-152 (2006).

10. Henderson, F. W., Collier, A. M., Clyde, W. A. Jr. \& Denny, F. W. N. Engl. J. Med. 300, 530-534 (1979).

11. Glezen, W. P., Taber, L. H., Frank, A. L. \& Kasel, J. A. Am. J. Dis. Child. 140, 543-546 (1986).

\section{The need for ethical guidance for the use of patient-reported outcomes in research and clinical practice}

To the Editor-Patient-reported outcomes (PROs) are increasingly being used in clinical research to provide evidence of the benefits and risk of therapy from a patient perspective. PRO data from clinical trials can inform regulatory approvals and drug labeling, clinical guideline development and health policy ${ }^{1}$. Approximately one third of clinical trials include PROs collected through the use of patient questionnaires $^{2}$. Beyond trials, PRO data are also increasingly captured in observational research and routine clinical care to provide information on the burden of disease and real-world evidence of treatment safety and effectiveness ${ }^{3}$, for audit and benchmarking ${ }^{1}$, and to monitor the status of patients and provide timely care tailored to individual needs. For instance, a study demonstrated that systematic web-based collection of information on symptoms led to improved health-related quality of life, survival and quality-adjusted survival, and fewer visits to the emergency room and hospitalization, among patients receiving chemotherapy for advanced solid tumors ${ }^{4}$. Patients value PRO trial results, as they can enhance clinicianpatient communication about treatment options, which helps patients to feel more empowered in shared decision-making around their care ${ }^{5}$.

Despite the benefits of incorporating PROs in research and routine practice, several ethical challenges can hinder the uptake and benefit to patients of PRO data. The PRO content of trial protocols and reporting of $\mathrm{PRO}$ results are often suboptimal, missing data rates are high, and delay of the publication of PRO data is commonplace. A recent study evaluating 228 studies from the National Institute of Health Research Cancer Portfolio demonstrated that 50,000 patients were involved in studies that failed to publish the PRO data collected, which is considered unethical ${ }^{6}$.

PRO data collection is associated with a number of ethical considerations that must be addressed. An ethical consideration is defined as one that requires a choice based on moral considerations drawing on established principles, theories and values, that might have implications for the person's or society's welfare. The differing use of PROs in research and routine care settings, and review and/or use of data by clinical teams, may lead to uncertainties for patients about why data are being collected and data privacy-how their data are being viewed and used. Research indicates that in some instances, PRO measures may not reflect the perspectives of vulnerable groups or older people, which challenges bioethical principles and threatens the scientific validity of results ${ }^{7}$. The burden on patients associated with the completion of multiple questionnaires is also a concern. Of particular note is the lack of guidance on how staff should manage situations in which PRO data reveal 'concerning' levels of psychological distress or physical symptoms that may require an immediate response . $^{8}$. Evidence suggests research staff are handling such data inconsistently, which may lead to inequitable patient care, co-intervention bias and confusion.

Furthermore, PROs could be used for long-term follow-up to assess the impact of the coronavirus SARS-CoV-2 on patients' quality of life and alert clinicians of potential life-threating symptoms ${ }^{9}$. The increased use of telehealth will also heighten the use of PRO data to monitor patients' symptoms. Therefore, there is a need to ensure that this is done in an ethical way that protects patients' safety and data.

To address these challenges, the PRO Ethics Steering Group, composed of PRO methodologists, patient partners 